\title{
A GENERAL PROCEDURE FOR DERIVING SYMMETRIC EXPRESSIONS FOR THE SECANT AND TANGENT STIFFNESS MATRICES IN FINITE ELEMENT ANALYSIS
}

\author{
ANTONIO MORÁN ${ }^{1}$, EUGENIO OÑATE ${ }^{2, *}$ AND JUAN MIQUEL ${ }^{2}$ \\ ${ }^{1}$ Dpto. Construcción e Ingeniería de Fabricación, Universidad de Oviedo, 33203 Gijón, Spain \\ ${ }^{2}$ E. T. S. Ingenieros de Caminos, Canales y Puertos, Universidad Politécnica de Cataluña, 08034 Barcelona, Spain
}

\begin{abstract}
The paper presents a general and straightforward procedure based on the use of the strain energy density for deriving symmetric expressions of the secant and tangent stiffness matrices for finite element analysis of geometrically non-linear structural problems. The analogy with previously proposed methods for deriving secant and tangent matrices is detailed. The simplicity of the approach is shown in an example of application. (C) 1998 John Wiley \& Sons, Ltd.
\end{abstract}

KEY WORDS: secant and tangent stiffness matrices; structural analysis; finite element method

\section{INTRODUCTION}

The possibilities of using the exact form of the secant stiffness matrix for developing new solution techniques for non-linear structural problems has been recently admitted by different authors. ${ }^{1-12}$ Particularly Kröplin and Dinkler ${ }^{6,7}$ and Oñate ${ }^{1,12}$ have used the concept of secant stiffness matrix successfully to propound new procedures for predicting the degree of structural instability and estimating limit and bifurcation points.

One of the problems in using secant stiffness matrix-based procedures is that the expression of this matrix is not unique and, therefore, unless some precautions are taken ${ }^{10,13}$ non-symmetric forms will be obtained. The successful applications reported in References 6, 7 and 12 have motivated the authors to explore new possibilities of formulating symmetric forms of the secant stiffness matrix for a larger variety of problems.

\section{Historical background}

In 1968, Mallett and Marcal analysed three different methods to solve a non-linear structural problem using the finite element method together with total Lagrangian formulation. ${ }^{8}$ The first formulation consisted in minimizing the functional representing the total potential energy of the structure. The second was based on writing the equilibrium equations in the deformed

\footnotetext{
* Correspondence to: Eugenio Oñate, International Centre for Numerical Methods in Engineering, Modulo C1, Campus Norte UPC, Gran Capital, s/n, 08034 Barcelona, Spain. E-mail: onate@etseccpb.upc.es 
configuration directly. The third method used an incremental approach, which consisted in gradually applying the external loads $\mathbf{f}$ in small load increments $\boldsymbol{\Delta f}$. At the same time the governing equations were linearized for each increment. Mallett and Marcal ${ }^{8}$ proved, by means of simple examples, that the three procedures lead to expressions of the type:

$$
\begin{aligned}
U & =\mathbf{a}^{\mathrm{T}} \mathbf{K}_{\mathrm{U}} \mathbf{a}, & \mathbf{K}_{\mathrm{U}} & =\frac{1}{2} \mathbf{K}_{0}+\frac{1}{6} \mathbf{N}_{1}+\frac{1}{12} \mathbf{N}_{2} \\
\mathbf{f} & =\mathbf{K}_{\mathrm{S}} \mathbf{a}, & & \mathbf{K}_{\mathrm{S}}=\mathbf{K}_{0}+\frac{1}{2} \mathbf{N}_{1}+\frac{1}{3} \mathbf{N}_{2} \\
\Delta \mathbf{f} & =\mathbf{K}_{\mathrm{T}} \Delta \mathbf{a}, & & \mathbf{K}_{\mathrm{T}}=\mathbf{K}_{0}+\mathbf{N}_{1}+\mathbf{N}_{2}
\end{aligned}
$$

where $U$ is the total strain energy function, $\mathbf{a}$ and $\Delta \mathbf{a}$ the nodal displacement and displacement increment vectors, respectively. $\mathbf{K}_{\mathrm{U}}, \mathbf{K}_{\mathrm{S}}$ and $\mathbf{K}_{\mathrm{T}}$, respectively, represent the energy, secant and tangent stiffness matrices of the system. Finally $\mathbf{K}_{0}, \mathbf{N}_{1}$ and $\mathbf{N}_{2}$ are three basic matrices whose combination provides the first three stiffness matrices, as shown in equation (1).

Rajasekaran and Murray ${ }^{13}$ noticed, some years later, that only certain decompositions of $\mathbf{K}_{\mathrm{U}}$ - see equation (1a) - ensure the derivation as given by equations (1b) and (1c). In order to justify the coefficients of $\mathbf{K}_{0}, \mathbf{N}_{1}$ and $\mathbf{N}_{2}$ in equations (1a) and (1c), these authors pointed out that each of the basic matrices was formed by equal degree terms in the unknown displacements. $\mathbf{K}_{0}$ was formed by constant term (zero degree), $\mathbf{N}_{1}$ by linear terms and $\mathbf{N}_{2}$ by quadratic terms. In an effort to preserve the formal relations between expressions (1a)-(1c), the same authors proposed
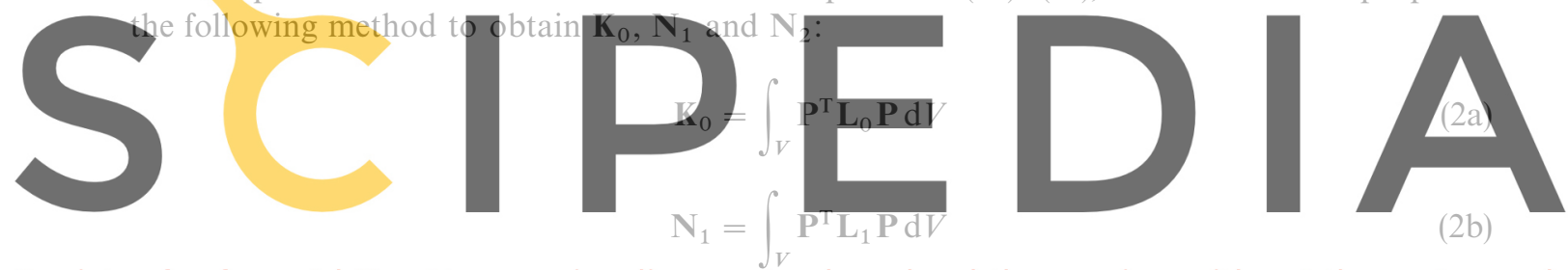

Register for free at https//www.scipedia.com to download the version without the watermark

$$
\mathbb{N}_{2}=\int_{V} \mathbb{P}^{\mathrm{T}} \mathbb{L}_{2} \mathbb{P} d V
$$

with

$$
\begin{aligned}
& \mathbf{L}_{0}=C_{i j} \mathbf{h}_{i} \mathbf{h}_{j}^{\mathrm{T}} \\
& \mathbf{L}_{1}=C_{i j}\left[\mathbf{h}_{i} \mathbf{g}^{\mathrm{T}} \mathbf{H}_{j}+\left(\mathbf{g}^{\mathrm{T}} \mathbf{h}_{i}\right) \mathbf{H}_{j}+\mathbf{H}_{i} \mathbf{g h}{ }_{j}^{\mathrm{T}}\right] \\
& \mathbf{L}_{2}=C_{i j}\left[\mathbf{H}_{i} \mathbf{g g}^{\mathrm{T}} \mathbf{H}_{j}+\frac{1}{2}\left(\mathbf{g}^{\mathrm{T}} \mathbf{H}_{j} \mathbf{g}\right) \mathbf{H}_{i}\right]
\end{aligned}
$$

where $V$ denotes the element volume (in the unloaded configuration geometry for a total lagrangian description), $C_{i j}$ represents the elements of the elastic constitutive matrix $\mathbf{C}$. In turn, $\mathbf{h}_{i}$ and $\mathbf{H}_{i}$ are symmetric vectors and matrices obtained by expanding each strain component $\varepsilon_{i}$ in terms of the displacement gradient vector $\mathbf{g}$ as:

$$
\varepsilon_{i}=\mathbf{h}_{i}^{\mathrm{T}} \mathbf{g}+\frac{1}{2} \mathbf{g}^{\mathrm{T}} \mathbf{H}_{i} \mathbf{g}
$$

Finally $\mathbf{P}$ is the so-called gradient matrix which relates displacement gradients and nodal displacements:

$$
\mathbf{g}=\mathbf{P a}
$$


Working with specific elements Rajasekaran and Murray also showed that non-linear stiffness matrices are not uniquely determined and so, many alternative forms were found to be nonsymmetric. The generalization of this concept for arbitrary elements was partly done by Felippa in Reference 2 and later by Felippa ${ }^{3}$ and Felippa and Crivelli ${ }^{3,4}$ by introducing the so-called Core-Congruential Formulation (CCF). These authors obtained a parametric form of the energy and secant stiffness matrices in the context of a total lagrangian description, yielding the symmetric expressions originally derived by Mallett and Marcal, ${ }^{8}$ as a particular case. A recent detailed description of the CCF can be found in Reference 5.

An alternative parametric expression of the secant stiffness matrix for solids and trusses has been recently presented by Oñate ${ }^{11}$ using a generalized Lagrangian description.

Attempts to obtain 'attractive' expressions of the secant stiffness matrix have been proposed by different authors. Wood and Schrefler, ${ }^{14}$ for instance, reproduced the symmetric expressions (1a) and (1c) using the B-notation due to Zienkiewicz and co-workers. ${ }^{14,15}$ More recently Badawi and Cusens, ${ }^{16}$ using some of the ideas in Reference 8, reported a simpler expression of the form proposed by Wood and Schrefler. Carrera ${ }^{1}$ presented a particular and simple symmetric form of the secant stiffness matrix for beam and plate analysis. Alternative symmetric expressions of the secant stiffness matrix have been derived by Kröplin et al. ${ }^{2,6,7}$ using a mixed formulation.

This paper revisits the ideas behind those of Felippa, Rajasekaran and Murray in order to derive a general symmetric form of both the secant and tangent matrices. The basic concept is the expansion of the strain energy density as the sum of homogeneous functions in unknown displacements. The secant and tangent matrices are simply obtained by adequate computation of
the first-and second-order derivatives of the total potential energy with respect to the nodal
displacements. Details of this procedure are given below.

DERIVATION OF THE SECANT AND TANGENT STIFFNESS MATRICES Register for free at https//wWW.SCipedia.COM to downioad the VETSton without the watermark Let us consider a structural system composed by a hyperelastic material. The generalized stress vector $\sigma_{\mathrm{g}}$ is obtained from the standard expression

$$
\boldsymbol{\sigma}_{\mathrm{g}}=\partial \Psi\left(\boldsymbol{\varepsilon}_{\mathrm{g}}\right) / \partial \boldsymbol{\varepsilon}_{\mathrm{g}}
$$

where $\Psi$ is the strain energy density and $\varepsilon_{\mathrm{g}}$ a generalized strain vector. Depending on the nature of the system, a structure or a solid, the vector $\boldsymbol{\sigma}_{\mathrm{g}}$ will represent a set of internal forces or stresses.

Let us suppose the system geometry defined by a set of $l$ kinematic variables $u_{i}$. In a Total Lagrangian Formulation (TL) the components of the vector $\boldsymbol{\varepsilon}_{\mathrm{g}}$ will depend on $m$ strain functions $p_{i}$, and these, in turn, will depend on the kinematic variables $u_{i}$ through some functional relations, i.e.:

$$
\varepsilon_{\mathrm{g}}=\varepsilon_{\mathrm{g}}[\mathbf{p}(\mathbf{u})]
$$

Functions $p_{i}$ and variables $u_{i}$ have been grouped in vectors $\mathbf{p}$ and $\mathbf{u}$

$$
\mathbf{p}^{\mathrm{T}}:=\left(p_{1}, p_{2}, \ldots, p_{m}\right), \quad \mathbf{u}^{\mathrm{T}}:=\left(u_{1}, u_{2}, \ldots, u_{l}\right)
$$

In solid continuous media, $\mathbf{p}(\mathbf{u})$-functions are usually identified with the components of the Jacobian matrix $\mathbf{J}: \mathbf{p}=\left\{\ldots \partial u_{i} / \partial x_{j} \ldots\right\}$. For more general systems, they may be arbitrary functions of the kinematic variables $u_{i}$ and their $n$-order derivatives $\partial^{n} u_{i} / \partial x_{j} \ldots \partial x_{k}$. 
The total strain energy $U$ is a TL formulation is obtained by integrating $\Psi$ over the reference configuration volume:

$$
U=\int_{V} \Psi \mathrm{d} V
$$

Assuming that the system is loaded by a set of external forces $\mathbf{F}$, equilibrium at an arbitrary deformed configuration, implies satisfaction of the principle of virtual work:

$$
\delta U=\int_{V} \sigma_{\mathrm{g}}^{\mathrm{T}} \delta \varepsilon_{\mathrm{g}} \mathrm{d} V=\int_{V} \mathbf{F}^{\mathrm{T}} \delta \mathbf{v} \mathrm{d} V
$$

where $\delta \mathbf{v}$ and $\mathbf{F}$ are two conjugate vectors of kinematically admissible virtual displacements and external loads respectively. In general, $\delta \mathbf{v}$ and $\mathbf{F}$ depend on the kinematic vector $\mathbf{u}$. On the other hand, for quasi-static analysis processes, external loads are applied gradually and we could write:

$$
\mathbf{F}=\lambda \overline{\mathbf{F}}[\mathbf{u}], \quad \delta \mathbf{v}=\mathbf{T}[\mathbf{u}] \delta \mathbf{u}
$$

where $\mathbf{T}$ is an $l$-order matrix which transforms the virtual kinematic vector $\delta \mathbf{u}$ into the virtual displacement vector $\delta \mathbf{v}$, and $\lambda$ is a loading parameter ranging from 0 to 1 .

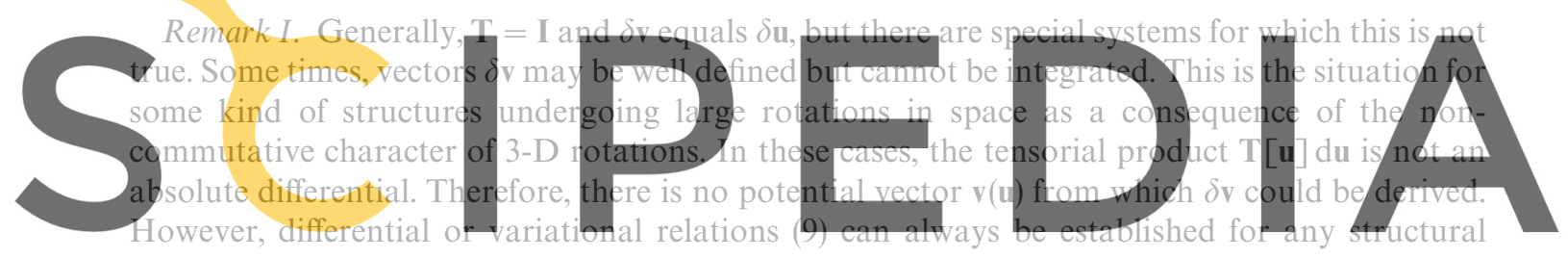

system.

Register for free at https//www.scipedia.com to download the version without the watermark Relations (5)-(9) yield

$$
\delta U=\int_{V} \frac{\partial \Psi}{\partial \mathbf{p}} \delta \mathbf{p} \mathrm{d} V=\int_{V} \mathbf{F}^{\mathrm{T}} \mathbf{T} \delta \mathbf{u} \mathrm{d} V
$$

For a conservative system the right-hand side term of equation (10) can be written as

$$
\int_{V} \mathbf{F}^{\mathrm{T}} \mathbf{T} \delta \mathbf{u} \mathrm{d} V=\int_{V} \delta \Omega \mathrm{d} V=\delta W
$$

And the total potential energy function is obtained as

$$
\Phi=U-W=\int_{V}(\Psi-\Omega) \mathrm{d} V
$$

Subdividing the structural system into finite elements and, assuming a standard interpolation for the kinematic variables $u_{i}$, the strain functions $p_{i}$ may be expressed in terms of nodal displacements, $a_{1}, \ldots, a_{n}$, as $p_{i}=p_{i}\left[a_{j}\right]$. Obviously, functions $p_{i}$ also depend on the finite element intepolation chosen, that is, $p_{i}=p_{i}\left(a_{j}, N_{j}, \partial N_{j} / \partial x_{k}, \ldots\right)$ where $N_{j}$ represents the interpolation shape functions. The variations in $u_{i}$ variables and $p_{i}$ functions, associated with virtual 
nodal displacements, can be written in matrix form as

$$
\delta \mathbf{u}=\mathbf{N} \delta \mathbf{a}, \quad \delta \mathbf{p}=\mathbf{P}[\mathbf{a}] \delta \mathbf{a}
$$

where $\mathbf{a}=\left[a_{1}, a_{2}, \ldots, a_{n}\right]^{\mathrm{T}}, \mathbf{P}$ is an $m \times n$ transformation matrix and $\mathbf{N}$ is the classical $l \times n$ shape function matrix. Introduction of equation (13) into the governing virtual work relationship of equation (11) yields:

$$
\delta U=\int_{V} \frac{\partial \Psi}{\partial \mathbf{p}} \mathbf{P} \delta \mathbf{a} \mathrm{d} V=\int_{V} \mathbf{F}^{\mathrm{T}} \mathbf{T} \mathbf{N} \delta \mathbf{a} \mathrm{d} V
$$

Satisfaction of equation (14) in the presence of non-trivial virtual displacements requires

$$
\int_{V} \frac{\partial \Psi}{\partial \mathbf{p}} \mathbf{P} \mathrm{d} V=\int_{V} \mathbf{F}^{\mathrm{T}} \mathbf{T} \mathbf{N} \mathrm{d} V=\mathbf{f}^{\mathrm{T}}
$$

where $\mathbf{f}$ is the vector of equivalent nodal forces.

Performing a second partial differentiation in (15), the linear relationship between differential increments of nodal displacements and forces can be obtained:

$$
\left[\int_{V} \mathbb{P}^{\mathrm{T}} \frac{\partial^{2} \Psi}{\partial \mathbf{p} \partial \mathbf{p}} \mathbf{P}+\frac{\partial \Psi}{\partial \mathbf{p}} \frac{\partial \mathbf{P}}{\partial \mathbf{a}} \mathrm{d} V\right] \mathrm{d} \mathbf{a}=\frac{\partial \mathbf{f}}{\partial \mathbf{a}} \mathrm{d} \mathbf{a}=\mathrm{d} \mathbf{f}
$$
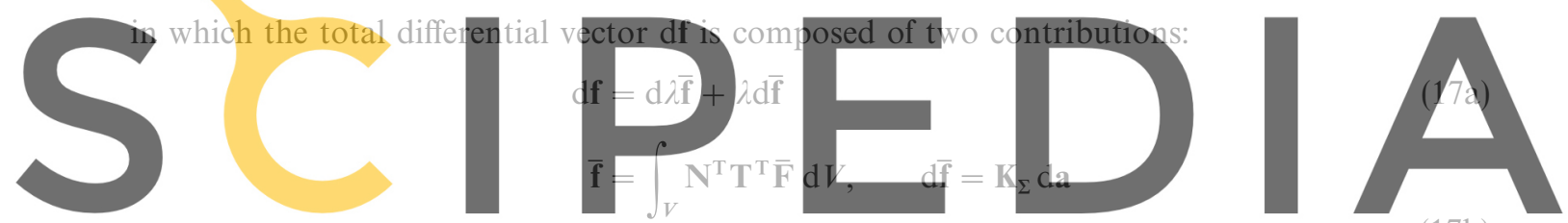

(17b)

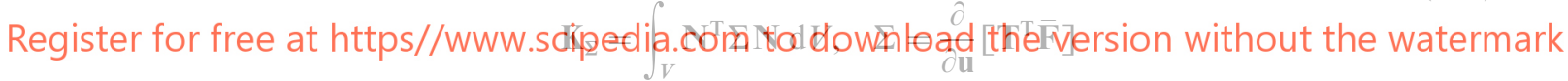

The first term in (17a) arises from an intensity change of the load external field $\mathbf{F}$. The second term arises from the change of the system geometry. When the load field is not conservative, $\mathbf{K}_{\Sigma}$ is a non-symmetrical matrix. Conversely, if the external load field is conservative, equation (11) holds, $\overline{\mathbf{F}}^{\mathrm{T}} \mathbf{T}$ du represents the absolute differential of a scalar field $\Omega$ and $\mathbf{K}_{\Sigma}$ is symmetrical:

$$
\overline{\mathbf{F}}^{\mathrm{T}} \mathbf{T} \mathrm{d} \mathbf{u}=\frac{\partial \Omega}{\partial \mathbf{u}} \mathrm{d} \mathbf{u}, \quad \Sigma(i, j)=\frac{\partial^{2} \Omega}{\partial u_{i} \partial u_{j}}
$$

Generally, $\mathbf{T}$ coincides with the identity matrix and $p_{i}$ functions are linear on $a_{i}$, that is, matrix $\mathbf{P}$ does not depend on $\mathbf{a}$ :

$$
\mathbf{T}=\mathbf{I}, \quad \mathbf{p}=\mathbf{P a}
$$

Assuming (19), expressions (16) and (17) are notably simplified:

$$
\begin{aligned}
& {\left[\int_{V} \mathbf{P}^{\mathrm{T}} \frac{\partial^{2} \Psi}{\partial \mathbf{p} \partial \mathbf{p}} \mathbf{P} \mathrm{d} V\right] \mathrm{d} \mathbf{a}=\mathrm{d} \lambda \overline{\mathbf{f}}+\lambda \mathbf{K}_{\Sigma} \mathrm{d} \mathbf{a}} \\
& \overline{\mathbf{f}}=\int_{V} \mathbf{N}^{\mathrm{T}} \overline{\mathbf{F}} \mathrm{d} V, \quad \mathbf{K}_{\Sigma}=\int_{V} \mathbf{N}^{\mathrm{T}} \frac{\partial \overline{\mathbf{F}}}{\partial \mathbf{u}} \mathbf{N} \mathrm{d} V
\end{aligned}
$$


In this case, the general matrix expressions of force-balance equilibrium equations and linear relationship between differential increments of nodal displacements and forces yield from (15) and (20):

$$
\begin{aligned}
\mathbf{f} & =\mathbf{K}_{\mathrm{S}} \mathbf{a} \\
\mathrm{d} \lambda \overline{\mathbf{f}} & =\left[\mathbf{K}_{\mathrm{T}}-\lambda \mathbf{K}_{\mathbf{\Sigma}}\right] \mathrm{d} \mathbf{a} \\
\mathbf{K}_{\mathrm{S}}[\mathbf{a}] \mathbf{a} & =\int_{V} \frac{\partial \Psi}{\partial \mathbf{p}}[\mathbf{a}] \mathbf{P} \mathrm{d} V \\
\mathbf{K}_{\mathrm{T}}[\mathbf{a}] & =\int_{V} \mathbf{p}^{\mathrm{T}} \frac{\partial^{2} \Psi}{\partial \mathbf{p} \partial \mathbf{p}}[\mathbf{a}] \mathbf{P} \mathrm{d} V
\end{aligned}
$$

where $\mathbf{K}_{\mathrm{S}}$ and $\mathbf{K}_{\mathrm{T}}$ are the secant and tangent global stiffness matrices for hyperelastic systems which have an indirect functional dependence on nodal displacements a.

In practice, as it is well known, global matrices are obtained assembling a set of local stiffness matrices, obtained independently for each element. Assuming the linearity condition (19), a systematic procedure to obtain these local stiffness matrices is presented in the next section. As in this article we are only interested in the decomposition of $\mathbf{K}_{\mathrm{U}}$, $\mathbf{K}_{\mathrm{S}}$ and $\mathbf{K}_{\mathrm{T}}$ matrices, we will also assume the contribution of the $\mathbf{K}_{\Sigma}$ matrix in equation (22a) as null.

Moreover, in order to simplify the notation, from now onwards, the first-, second- and hy gher-order partial derivatives of any function $g$ will be denote the domain of definition of an element. 12 HOMOGENEOUS DECOMPOSITIO DERIVATION OF

THESECANT AND TANGENT
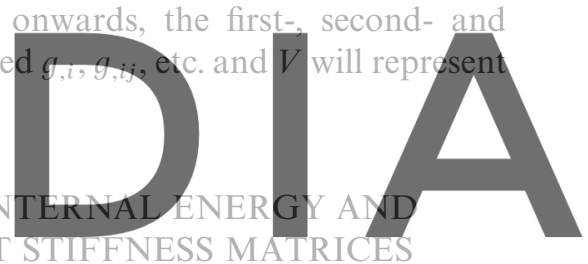

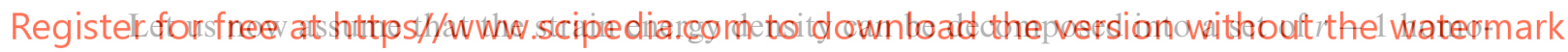
geneous functions on $p_{i}$ variables of degree $n=2,3, \ldots, r$, such that

$$
\Psi\left(p_{i}\right)=\Psi^{(2)}\left(p_{i}\right)+\Psi^{(3)}\left(p_{i}\right)+\cdots+\Psi^{(r)}\left(p_{i}\right)
$$

This assumption is satisfied by most known structural systems. Taking into account the general properties of homogeneous functions of $n$th degree, each component $\Psi^{(n)}$ of $\Psi$ and their first partial derivatives $\Psi_{, i}^{(n)}$ could be expressed in terms of their second-order partial derivatives $\Psi_{, i j}^{(n)}$ (Euler's theorem) as follows:

$$
\begin{aligned}
& \Psi^{(n)} \equiv \frac{1}{n(n-1)} \Psi_{, i j}^{(n)} p_{i} p_{j} \\
& \Psi_{, i}^{(n)} \equiv \frac{1}{(n-1)} \Psi_{, i j}^{(n)} p_{j}
\end{aligned}
$$

Introducing (24) into (7) and (22), the internal energy function $U$ and the secant and tangent stiffness matrices can be decomposed and expanded into a set of basic matrices $\mathbf{B}$ :

$$
\begin{aligned}
U & =\mathbf{a}^{\mathrm{T}} \mathbf{K}_{\mathrm{U}} \mathbf{a} \\
\mathbf{f} & =\mathbf{K}_{\mathrm{S}} \mathbf{a} \\
\mathrm{d} \mathbf{f} & =\mathbf{K}_{\mathrm{T}} \mathrm{d} \mathbf{a}
\end{aligned}
$$




$$
\begin{aligned}
& \mathbf{K}_{\mathrm{U}}=\int_{V} \mathbf{P}^{\mathrm{T}}\left[\frac{1}{2} \mathbf{B}^{(2)}+\frac{1}{6} \mathbf{B}^{(3)}+\frac{1}{12} \mathbf{B}^{(4)}+\cdots+\frac{1}{r(r-1)} \mathbf{B}^{(r)}\right] \mathbf{P} \mathrm{d} V \\
& \mathbf{K}_{\mathrm{S}}=\int_{V} \mathbf{P}^{\mathrm{T}}\left[\mathbf{B}^{(2)}+\frac{1}{2} \mathbf{B}^{(3)}+\frac{1}{3} \mathbf{B}^{(4)}+\cdots+\frac{1}{(r-1)} \mathbf{B}^{(r)}\right] \mathbf{P} \mathrm{d} V \\
& \mathbf{K}_{\mathrm{T}}=\int_{V} \mathbf{P}^{\mathrm{T}}\left[\mathbf{B}^{(2)}+\mathbf{B}^{(3)}+\mathbf{B}^{(4)}+\cdots+\mathbf{B}^{(r)}\right] \mathbf{P} \mathrm{d} V
\end{aligned}
$$

$\mathbf{B}^{(n)}$ matrices are defined as follows:

$$
\mathbf{B}^{(n)}(i, j)=\Psi_{, i j}^{(n)}
$$

Notice the nature of $\mathbf{B}^{(n)}$ matrices repeated in the three expressions (26). Naturally, these $\mathbb{B}$ matrices are functions of the $p_{i}$ variables.

From the previous definition it can be inferred that all $\mathbf{B}^{(2)}, \mathbf{B}^{(3)}, \ldots, \mathbf{B}^{(r)}$ matrices are symmetric and homogeneous of degrees $0,1, \ldots,(r-2)$, respectively. Furthermore, in the particular and relatively common case of $r$ being equal to 4, comparing equations (26) and (1a), the equivalence between the result obtained by Mallett and $\mathrm{Marcel}^{8}$ and this procedure is obvious:
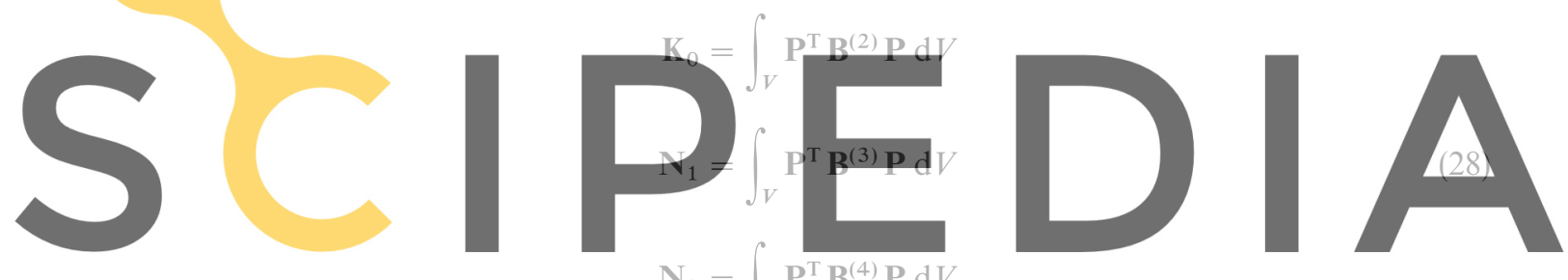

Register for free at https//www.scipedia.com to download the version without the watermark

Moreover, comparing equations (28) and (2), the relationship between $\mathbb{B}^{(n)}$ matrices and the matrices deduced by Rajasenkaran and Murray (see equations (2) and (3)) yields:

$$
\mathbf{I}_{0}=\mathbf{B}^{(2)}, \quad \mathbf{I}_{1}=\mathbf{B}^{(3)}, \quad \mathbf{I}_{2}=\mathbf{B}^{(4)}
$$

The analogy between the three procedures is now complete. One of the most interesting properties which follows from (26), and from the homogeneous character of $\mathbf{B}$ matrices, is the possibility of expressing the internal energy and secant and tangent stiffness matrices by simple polynomial expansions in $\lambda$, when moving in a specified direction $\mathbf{a}=\lambda \mathbf{z}$ :

where

$$
U(\mathbf{a})=\sum_{n=2}^{r} \lambda^{n} u(\mathbf{z}), \quad \mathbf{f}(\mathbf{a})=\sum_{n=2}^{r} \lambda^{(n-1)} f_{n}(\mathbf{z}), \quad \mathbf{K}_{\mathrm{T}}(\mathbf{a})=\sum_{n=2}^{r} \lambda^{(n-2)} \mathbf{K}_{n}(\mathbf{z})
$$

$$
\mathbf{K}_{n}(\mathbf{z})=\int_{V} \mathbf{P}^{\mathrm{T}} \mathbf{B}^{(n)}(\mathbf{z}) \mathbf{P} \mathrm{d} V, \quad f_{n}(\mathbf{z})=\frac{1}{n-1} \mathbf{K}_{n}(\mathbf{z}) \mathbf{z}, \quad u_{n}(\mathbf{z})=\frac{1}{n(n-1)} \mathbf{z}^{\mathrm{T}} \mathbf{K}_{n}(\mathbf{z}) \mathbf{z}
$$

This property has interesting applications to structural stability problems, and it has been recently used by Oñate and his coworkers ${ }^{11,12}$ to calculate critical loads and bifurcation points in structures. 
The efficient calculation of $\mathbf{B}^{(n)}$ matrices requires a detailed knowledge of the specific form of the strain-energy function $\Psi$. Generally, this function can be written as follows:

$$
\Psi=\frac{1}{2} \varepsilon_{\mathrm{g}}^{\mathrm{T}} \mathbf{C}\left(\boldsymbol{\varepsilon}_{\mathrm{g}}\right) \boldsymbol{\varepsilon}_{\mathrm{g}}
$$

where $\mathbf{C}$ is a symmetric constitutive matrix and $\boldsymbol{\varepsilon}_{\mathrm{g}}$ represents the generalized deformation vector (for simplicity, the ' $\mathrm{g}$ ' index of $\boldsymbol{\varepsilon}$ will be omitted in the sequel).

In most cases, $\mathbf{C}$ is a constant matrix and vector $\boldsymbol{\varepsilon}$ depends on $p_{i}$ functions through a polynomial expression which can be written as:

$$
\boldsymbol{\varepsilon}\left(p_{i}\right)=\boldsymbol{\varepsilon}^{(1)}\left(p_{i}\right)+\boldsymbol{\varepsilon}^{(2)}\left(p_{i}\right)+\cdots+\boldsymbol{\varepsilon}^{(s)}\left(p_{i}\right)
$$

where $\boldsymbol{\varepsilon}^{(k)}$ contains all $k$-order products on $p_{i}$ functions, i.e. $\boldsymbol{\varepsilon}^{(k)}$ are also homogeneous polynomial functions of $k$ th degree.

If this is the case, the $\Psi^{(n)}$ components of the strain energy density function which appear in equation (23), may be calculated as:

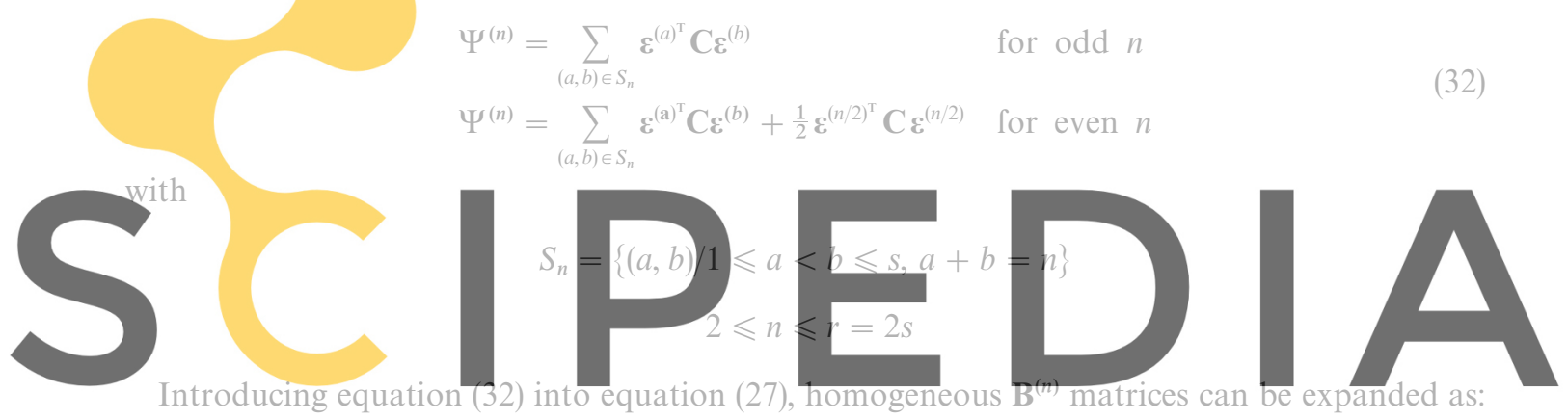

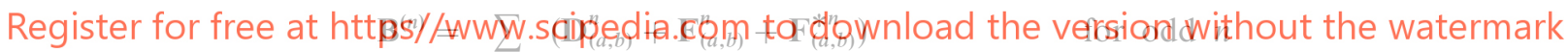

$$
\mathbf{B}^{(n)}=\sum_{(a, b) \in S_{n}}\left(\mathbf{D}_{(a, b)}^{n}+\mathbf{F}_{(a, b)}^{n}+\mathbf{F}_{(a, b)}^{* n}\right)+\mathbf{G}^{n}+\mathbf{G}^{* n} \text { for even } n
$$

In (33) $\left\{\mathbf{D} \ldots \mathbf{F}^{*}\right\}_{(a, b)}^{n}$ is a set of homogeneous and symmetric matrices of $n$th degree, associated to each pair of values $(a, b) \in S_{n}$, and defined as follows:

$$
\begin{aligned}
\mathbf{D}_{(a, b)}^{n} & =\mathbf{E}^{(a)^{\mathrm{T}}} \mathbf{C} \mathbf{E}^{(b)}+\mathbf{E}^{(b)^{\mathrm{T}}} \mathbf{C} \mathbf{E}^{(a)} \\
\mathbf{G}^{n} & =\mathbf{E}^{(n / 2)^{\mathrm{T}}} \mathbf{C} \mathbf{E}^{(n / 2)} \\
\mathbf{G}^{* n}(i, j) & =\boldsymbol{\sigma}^{(n / 2)^{\mathrm{T}}} \boldsymbol{\varepsilon}_{, i j}^{(n / 2)} \\
\mathbf{F}_{(a, b)}^{n}(i, j) & =\boldsymbol{\varepsilon}_{, i j}^{(a)^{\mathrm{T}}} \boldsymbol{\sigma}^{(b)} \\
\mathbf{F}_{(a, b)}^{* n}(i, j) & =\boldsymbol{\sigma}^{(a)^{\mathrm{T}}} \boldsymbol{\varepsilon}_{, i j}^{(b)}
\end{aligned}
$$

with

$$
\mathbf{E}^{(\cdot)}(i, j):=\boldsymbol{\varepsilon}_{, j}^{(\cdot)}(i) \quad \text { and } \quad \sigma^{(\cdot)}:=\mathbf{C} \boldsymbol{\varepsilon}^{(\cdot)}
$$

where $(\cdot)$ is introduced as an abbreviation for $(a)$ and $(b)$. Note that the right-hand side terms in equations $(34 \mathrm{c})-(34 \mathrm{e})$ are scalar products of two vectors. 
Some of these matrices are null. For example, $\mathbf{F}^{(n)}$ is always cancelled out when $a=1$. As a rule, the maximum number of different and no-null matrices of type (34), which have to be calculated to construct $\mathbf{B}^{(n)}$ matrices, is equal to $1 / 2 s(3 s-1)$. This number increases, therefore, with the square of the degree of polynomial $\varepsilon\left(p_{i}\right)$. As the complexity of the problem grows, the value of the variable $s$ also increases and so does the number of matrices needed to make decompositions (26) and (34). This can produce great storage problems in the computer's memory and may require a lot of calculation time to construct and assemble all those matrices. In these cases, the previous procedure can be simplified by assembling some terms of expression (31) previously homogenized, as we will see later, or by using a series of relations which simplify the calculation of both strain energy $U$ and secant stiffness matrix $\mathbf{K}_{\mathbf{S}}$. These relations can be obtained by a straightforward application of Euler's theorem on homogeneous functions.

In fact, as $\varepsilon^{(n)}$ and $\varepsilon_{, i}^{(n)}$ are homogeneous in degrees $n$ and $n-1$, we have

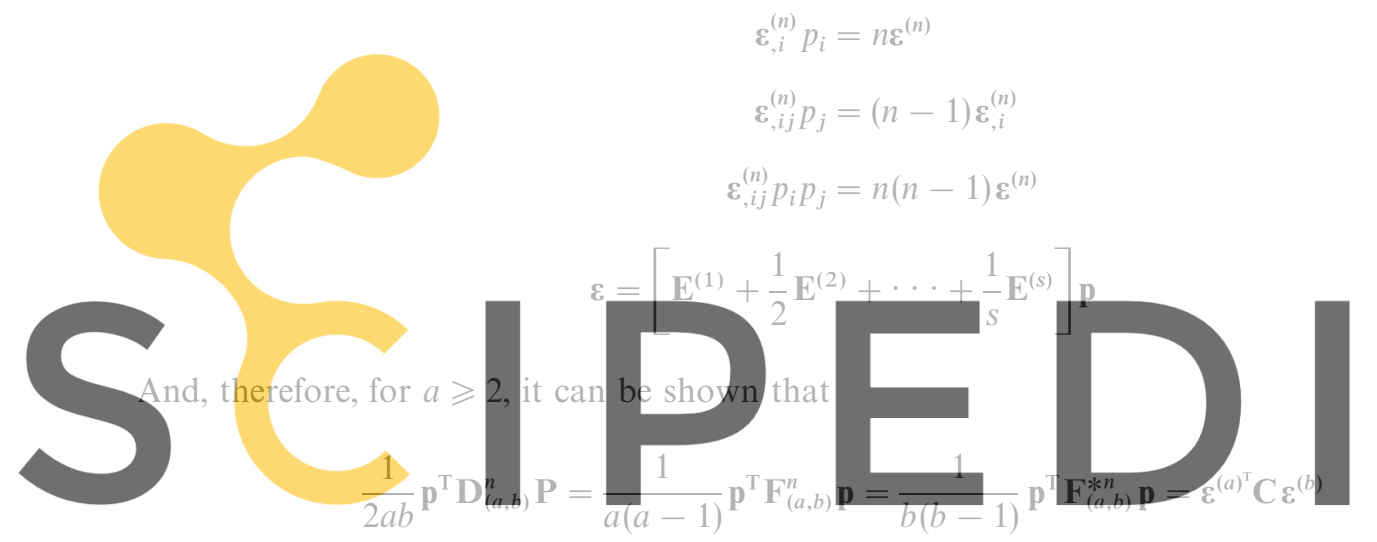

Register for free at https//www.scipedia.com to download the version without the watermark

$$
\begin{aligned}
\frac{4}{n^{2}} \mathrm{p}^{\mathrm{T}} \mathrm{G}^{n} \mathrm{p} & =\frac{4}{n(n-2)} \mathrm{p}^{\mathrm{T}} \mathrm{G}^{* n} \mathrm{p}=\varepsilon^{(n / 2)^{\mathrm{T}}} \mathrm{C} \varepsilon^{(n / 2)} \\
\mathbf{D}_{(a, b)}^{n} \mathbf{p} & =\frac{b}{(a-1)} \mathbf{F}_{(a, b)}^{n} \mathbf{p}+\frac{a}{(b-1)} \mathbf{F}_{(a, b)}^{* n} \mathbf{p}=b \mathbf{E}^{(a)^{\mathrm{T}}} \boldsymbol{\sigma}^{(b)}+a \mathbf{E}^{(b)^{\mathrm{T}}} \boldsymbol{\sigma}^{(a)} \\
\frac{2}{n} \mathbf{G}^{n} \mathbf{p} & =\frac{2}{(n-2)} \mathbf{G}^{* n} \mathbf{p}=\mathbf{E}^{(n / 2)^{\mathrm{T}}} \boldsymbol{\sigma}^{(n / 2)}
\end{aligned}
$$

For $a=1$, the four relations (38)-(39) hold if the terms in which the undetermined quotient between matrix $\mathbf{F}_{(1, b)}^{(n)}$ and factor $(a-1)$ appears are previously removed. The immediate conclusion which yields from (38) and (39) is that the result of decomposing (26) into a group of homogeneous $\mathbf{B}$ matrices is not unique, and that definition (33) of these matrices may be generalized to a wider family whose general expression for the internal energy matrix $\mathbf{K}_{\mathrm{U}}$ is:

$$
\mathbf{K}_{\mathbf{U}} \begin{cases}\mathbf{B}^{(n)}=\sum_{(a, b) \in S_{k}}\left(\alpha \mathbf{D}_{(a, b)}^{n}+\beta \mathbf{F}_{(a, b)}^{n}+\gamma \mathbf{F}_{(a, b)}^{* n}\right) & \text { for odd } n \\ \mathbf{B}^{(n)}=\sum_{(a, b) \in S_{k}}\left(\alpha \mathbf{D}_{(a, b)}^{n}+\beta \mathbf{F}_{(a, b)}^{n}+\gamma \mathbf{F}_{(a, b)}^{* n}\right)+\alpha^{\prime} \mathbf{G}^{n}+\beta^{\prime} \mathbf{G}^{* n} & \text { for even } n\end{cases}
$$


with the conditions

$$
\begin{array}{cl}
L_{1}(\alpha, \beta, \gamma)=L_{1}(1,1,1) & \text { if } a \geqslant 2 \\
L_{1}(\alpha, 0, \gamma)=L_{1}(1,0,1) & \text { if } a=1 \\
L_{2}\left(\alpha^{\prime}, \beta^{\prime}\right)=L_{2}(1,1) &
\end{array}
$$

and for the secant stiffness matrix $\mathbf{K}_{\mathrm{s}}$ :

$\mathbf{K}_{\mathbf{S}} \begin{cases}\mathbf{B}^{(n)}=\sum_{(a, b) \in S_{n}}\left[(1+\alpha(a-1)(b-1)) \mathbf{D}_{(a, b)}^{n}+(1-\alpha b(b-1)) \mathbf{F}_{(a, b)}^{n}+(1-\alpha a(a-1)) \mathbf{F}_{(a, b)}^{* n}\right. & \text { for odd } n \\ \mathbf{B}^{(n)}=\mathbf{B}^{(n)}(\operatorname{odd} n)+\alpha^{\prime} \mathbf{G}^{n}+\beta^{\prime} \mathbf{G}^{* n} & \text { for even } n\end{cases}$

\section{with the conditions}

$$
\left\{\begin{array}{l}
\alpha=0 \quad \text { if } a=1 \\
L_{2}\left(\alpha^{\prime}, \beta^{\prime}\right)=L_{2}(1,1)
\end{array}\right.
$$

In both cases, functions $L_{i}$ are defined as follows:

$$
L_{1}(\alpha, \beta, \gamma):=2 \alpha a b+\beta a(a-1)+\gamma b(b-1)
$$

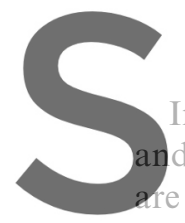

In the particular case in
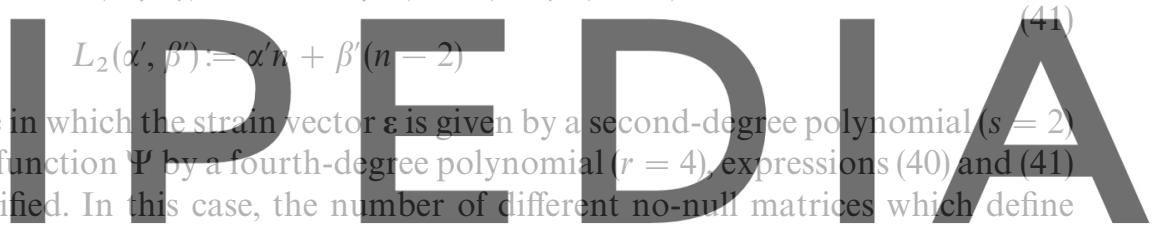

B matrices is equal to five; $S_{2}$ and $S_{4}$ are empty groups, and the only element of the group $S_{3}$ is the

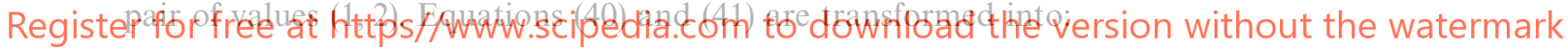

$$
\begin{aligned}
& \mathbb{B}^{(2)}=G^{(2)}
\end{aligned}
$$

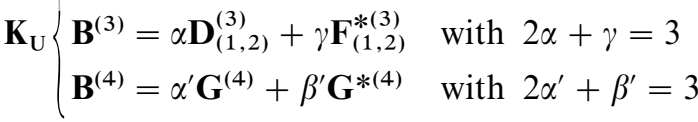

$$
\begin{aligned}
& \mathbf{K}_{\mathbf{S}}\left\{\begin{array}{l}
\mathbf{B}^{(2)}=\mathbf{G}^{(2)} \\
\mathbf{B}^{(3)}=\mathbf{D}_{(1,2)}^{(3)}+\mathbf{F}_{(1,2)}^{*(3)} \\
\mathbf{B}^{(4)}=\alpha^{\prime} \mathbf{G}^{(4)}+\beta^{\prime} \mathbf{G}^{*(4)} \quad \text { with } 2 \alpha^{\prime}+\beta^{\prime}=3
\end{array}\right.
\end{aligned}
$$

where $\mathbf{G}^{(2)}, \ldots, \mathbf{G}^{*(4)}$ matrices are defined in (34). If the deformation vector $\boldsymbol{\varepsilon}$ is also expressed in the usual way (4a), the expressions (34) become

$$
\begin{aligned}
\mathbf{G}^{(2)} & =C_{i j} \mathbf{h}_{i} \mathbf{h}_{j}^{\mathrm{T}} \\
\mathbf{D}_{(1,2)}^{(3)} & =C_{i j}\left[\mathbf{h}_{i} \mathbf{g}^{\mathrm{T}} \mathbf{H}_{j}+\mathbf{H}_{i} \mathbf{g} \mathbf{h}_{j}^{\mathrm{T}}\right] \\
\mathbf{F}_{(1,2)}^{*(3)} & =C_{i j}\left(\mathbf{g}^{\mathrm{T}} \mathbf{h}_{i}\right) \mathbf{H}_{j} \\
\mathbf{G}^{(4)} & =C_{i j} \mathbf{H}_{i} \mathbf{g} \mathbf{g}^{\mathrm{T}} \mathbf{H}_{j} \\
\mathbf{G}^{*(4)} & =\frac{1}{2} C_{i j}\left(\mathbf{g}^{\mathrm{T}} \mathbf{H}_{j} \mathbf{g}\right) \mathbf{H}_{i}
\end{aligned}
$$


By introducing (43) into (42) with $\alpha=\gamma=\alpha^{\prime}=\beta^{\prime}=1$, (26) yields relations (2) and (3) of Rajasekaran and Murray again. On the other hand, expressions (26) and (40) and (41) extend to $s>2$ the symmetrical forms that Felippa ${ }^{3}$ worked out for the special case (4a).

Remark 2. In practice (see Example 1), the manual calculation of basic $\mathbf{B}$ matrices can be more easily done by directly applying (27) than by getting help from a decomposition of the type (34) or by a pseudospectral development, like (42), especially if the problem is complex $(r>4)$. The value of the expanded series (33) and (34) or (42) and (43), is based on the possibility of programming the calculation of $\mathbf{B}$ matrices in such a way that a mathematical symbolic program could carry it out efficiently. Of course, B matrices can also be obtained calculating numerically the second derivatives of the functional $\Psi$, but the waste of computer time and associated roundoff errors advise against this procedure.

Remark 3. Some authors prefer deducing equilibrium equations and secant and tangent stiffness matrices from the principle of virtual work (PVW), instead of directly from the elastic deformation energy, as it has been done in this section. Even when the results are essentially the same, they are formally different, because, in each case, they are expressed in terms of a different group of basic matrices. The greatest objection to the method based on the PVW is that it does not ensure the symmetry of the secant stiffness matrix. Although for the particular case $s=2$ and $r=4^{11,14,16}$ symmetrical forms have been found, these are laborious and they cannot be generalized for other $s$ and $r$ values. However, a relationship can be established between both formulations. Let us prove it for $s=2, r=4$. In this case, introducing (19) into (37), the deformation vector can be written in terms of nodal variables a, as follows:

$$
\boldsymbol{\varepsilon}=\mathbf{B}_{0} \mathbf{a}+\frac{1}{2} \mathbf{B}_{\mathrm{L}}(\mathbf{a}) \mathbf{a} \text { and }\left\{\begin{array}{l}
\mathbf{B}_{0}=\mathbf{E}^{(1)} \mathbf{P} \\
\mathbf{B}_{\mathrm{L}}=\mathbf{E}^{(2)}(\mathbf{a}) \mathbf{P}
\end{array}\right.
$$

where the B-notation, characteristic of the methods based on the PVW, ${ }^{14,15}$ has been adopted.

With the following definitions:

$$
\mathbf{S}_{0}:=\boldsymbol{\sigma}^{(1)^{\mathrm{T}}} \boldsymbol{\varepsilon}_{, i j}^{(2)}, \quad \mathbf{S}_{\mathrm{L}}:=\boldsymbol{\sigma}^{(2)^{\mathrm{T}}} \boldsymbol{\varepsilon}_{, i j}^{(2)}
$$

and using (34), the following can be readily verified:

$$
\begin{aligned}
\mathbf{P}^{\mathrm{T}} \mathbf{G}^{(2)} \mathbf{P} & =\mathbf{B}_{0}^{\mathrm{T}} \mathbf{C} \mathbf{B}_{0} \\
\mathbf{P}^{\mathrm{T}} \mathbf{D}_{(1,2)}^{(3)} \mathbf{P} & =\mathbf{B}_{0}^{\mathrm{T}} \mathbf{C} \mathbf{B}_{\mathrm{L}}+\mathbf{B}_{\mathrm{L}}^{\mathrm{T}} \mathbf{C} \mathbf{B}_{0} \\
\mathbf{P}^{\mathrm{T}} \mathbf{F}_{(1,2)}^{*(3)} \mathbf{P} & =\mathbf{P}^{\mathrm{T}} \mathbf{S}_{0} \mathbf{P} \\
\mathbf{P}^{\mathrm{T}} \mathbf{G}^{(4)} \mathbf{P} & =\mathbf{B}_{\mathrm{L}}^{\mathrm{T}} \mathbf{C} \mathbf{B}_{\mathrm{L}} \\
\mathbf{P}^{\mathrm{T}} \mathbf{G}^{*(4)} \mathbf{P} & =\mathbf{P}^{\mathrm{T}} \mathbf{S}_{\mathrm{L}} \mathbf{P}
\end{aligned}
$$


From (26), (42) and (46) matrices $K_{0}, \mathbf{N}_{1}$ and $\mathbf{N}_{2}$, derived by Rajasekaran and Murray-equation (2)-are obtained as:

$$
\begin{aligned}
& \mathbf{K}_{0}=\int_{V} \mathbf{B}_{0}^{\mathrm{T}} \mathbf{C} \mathbf{B}_{0} \mathrm{~d} V \\
& \mathbf{N}_{1}=\int_{V}\left[\alpha\left(\mathbf{B}_{0}^{\mathrm{T}} \mathbf{C B}_{\mathrm{L}}+\mathbf{B}_{\mathrm{L}}^{\mathrm{T}} \mathbf{C} \mathbf{B}_{0}\right)+\gamma \mathbf{P}^{\mathrm{T}} \mathbf{S}_{0} \mathbf{P}\right] \mathrm{d} V \quad\left\{\begin{array}{l}
\mathbf{K}_{\mathrm{U}} \text { requires } 2 \alpha+\gamma=3 \\
\mathbf{K}_{\mathrm{S}} \text { and } \mathbf{K}_{\mathrm{T}} \text { require } \alpha=\gamma=1
\end{array}\right. \\
& \mathbf{N}_{2}=\int_{V}\left[\alpha^{\prime} \mathbf{B}_{\mathrm{L}}^{\mathrm{T}} \mathbf{C B}_{\mathrm{L}}+\beta^{\prime} \mathbf{P}^{\mathrm{T}} \mathbf{S}_{\mathrm{L}} \mathbf{P}\right] \mathrm{d} V \quad\left\{\begin{array}{l}
\mathbf{K}_{\mathrm{U}} \text { and } \mathbf{K}_{\mathrm{S}} \text { require } 2 \alpha^{\prime}+\beta^{\prime}=3 \\
\mathbf{K}_{\mathrm{T}} \text { requires } \alpha^{\prime}=\beta^{\prime}=1
\end{array}\right.
\end{aligned}
$$

Setting $\alpha=\gamma=1$ and $\alpha^{\prime}=\beta^{\prime}=1$ in (47) leads to the matrices expressions $\mathbf{K}_{0}, \mathbf{N}_{1}$ and $\mathbf{N}_{2}$ obtained by Wood and Schrefler. ${ }^{14}$ Also, making $\alpha=\gamma=1, \alpha^{\prime}=3 / 2$ and $\beta^{\prime}=0$, the Badawi and Cusens correction for the $\mathbf{K}_{\mathrm{S}}$ matrix ${ }^{16}$ is obtained.

In connection with the formulation of the initial stress problem in structural systems for which the strain vector $\varepsilon$ is defined by (4a), Oñate ${ }^{11,12}$ has derived a group of general forms, symmetric and non-symmetric, which extend the above results. By requiring the initial stresses to vanish, Oñate's symmetrical forms coincide with (47).

\section{OTHER EXPRESSIONS OF THE SECANT AND TANGENT MATRICES}

One of the most interesting characteristics of the decomposition (26) is that it allows a unified formulation of the three basic methods of non-linear analysis to be derived: minimization of the functional $\Psi$, direct resolution of the non-linear equation system and approximation by means of linearized incremental techniques. Since the same group of basic B matrices appears in the three alternative formulations, a solution procedure embodying all three techniques can be easily developed.

The biggest difficulty a decomposition like (26) presents is the computation of a large number of $\mathbf{B}$ matrices when the $r$-degree of $\Psi$ is high. In such cases, the method mentioned loses efficiency and the number of basic $\mathbf{B}$ matrices must be reduced, although some of them lose their homogeneous character in $p_{i}$ variables.

In this section, a simple procedure to obtain this reduction is presented. It consists in homogenizing all or some of the terms of (31), by premultiplying them by an auxiliary variable $t$ :

$$
\boldsymbol{\varepsilon}\left(p_{i}\right)=t^{s-1} \boldsymbol{\varepsilon}^{(1)}\left(p_{i}\right)+t^{s-2} \boldsymbol{\varepsilon}^{(2)}\left(p_{i}\right)+\cdots+\boldsymbol{\varepsilon}^{(s)}\left(p_{i}\right)
$$

In order to return to the original expression (31), the $t$ variable is later replaced by the unit value. Applying this idea to the particular case in which $\mathbf{C}$ is constant, $s=2$, and $r=4$, we obtain

$$
\boldsymbol{\varepsilon}\left(p_{i}\right)=t \boldsymbol{\varepsilon}^{(1)}\left(p_{i}\right)+\boldsymbol{\varepsilon}^{(2)}\left(p_{i}\right) \quad \text { with } t=1
$$

The strain energy density will be determined by a unique term of expansion (23):

$$
\Psi^{(4)}=\frac{1}{2}\left[t^{2} \boldsymbol{\varepsilon}^{(1)^{\mathrm{T}}} \mathbf{C} \boldsymbol{\varepsilon}^{(1)}+2 t \boldsymbol{\varepsilon}^{(1)^{\mathrm{T}}} \mathbf{C} \boldsymbol{\varepsilon}^{(2)}+\boldsymbol{\varepsilon}^{(2)^{\mathrm{T}}} \mathbf{C} \boldsymbol{\varepsilon}^{(2)}\right]
$$


Decomposition (26) will be, then, reduced to a unique homogeneous no-null matrix $\hat{\mathbf{B}}^{(4)}$ of fourth-degree and $(m+1)$ th order, giving

$$
\begin{aligned}
\hat{\mathbf{K}}_{\mathrm{U}} & =\frac{1}{12} \int_{V} \hat{\mathbf{P}}^{\mathrm{T}} \hat{\mathbf{B}}^{(4)} \hat{\mathbf{P}} \mathrm{d} V \\
\hat{\mathbf{K}}_{\mathrm{S}} & =\frac{1}{3} \int_{V} \hat{\mathbf{P}}^{\mathrm{T}} \hat{\mathbf{B}}^{(4)} \hat{\mathbf{P}} \mathrm{d} V \\
\hat{\mathbf{K}}_{\mathrm{T}} & =\int_{V} \hat{\mathbf{P}}^{\mathrm{T}} \hat{\mathbf{B}}^{(4)} \hat{\mathbf{P}} \mathrm{d} V
\end{aligned}
$$

where the new nodal displacement vector $\hat{\mathbf{a}}$ and the strain functions vector $\hat{\mathbf{p}}$ are defined by

$$
\hat{\mathbf{p}}=\hat{\mathbf{P}} \hat{\mathbf{a}} \quad \text { with } \quad \hat{\mathbf{a}}^{\mathrm{T}}=\left[\mathbf{a}^{\mathrm{T}}, t\right] ; \quad \hat{\mathbf{p}}^{\mathrm{T}}=\left[\mathbf{p}^{\mathrm{T}}, t\right] ; \quad \hat{\mathbf{P}}=\left[\begin{array}{cc}
\mathbf{P} & \mathbf{0} \\
\mathbf{0} & 1
\end{array}\right]
$$

The core-stiffness matrix $\hat{\mathbf{B}}^{(4)}$ of the congruential transformation (51) is directly calculated from (27). Moreover, by separating the auxiliary variable $t$ from the rest of variables $p_{i}(i: 1, \ldots, m), \hat{\mathbf{B}}^{(4)}$ can be partitioned as follows:

$$
\begin{aligned}
\hat{\mathbf{B}}^{(4)} & =\left[\begin{array}{ll}
\mathbf{B}_{(i, j)} & \mathbf{B}_{(i, m+1)} \\
\operatorname{Sim} . & \mathbf{B}_{(m+1, m+1)}
\end{array}\right] \\
\hat{\mathbf{B}}^{(4)}(i, j)_{(1 \leqslant i, j \leqslant m)} & =t^{2} \mathbf{B}^{(2)}+t \mathbf{B}^{(3)}+\mathbf{B}^{(4)} \\
\left.\hat{\mathbf{B}}^{(4)}(i, m+1)_{(1 \leqslant i \leqslant m)}\right) & =\left[2 t \mathbf{B}^{(2)}+\frac{1}{2} \mathbf{B}^{(3)}\right] \mathbf{p} \\
\hat{\mathbf{B}}^{(4)}(m+1, m+1) & =\mathbf{p}^{\mathrm{T}} \mathbf{B}^{(2)} \mathbf{p}
\end{aligned}
$$

Replacing (53) in the third equation (51) and integrating, the tangent matrix $\hat{\mathbf{K}}_{\mathrm{T}}$ is obtained. Now, making $t=1$ and using the notation of equations (1), $\hat{\mathbf{K}}_{\mathrm{T}}$ may be written as

$$
\hat{\mathbf{K}}_{\mathrm{T}}[\mathbf{a}]=\left[\begin{array}{c|c}
\mathbf{K}_{0}+\mathbf{N}_{1}+\mathbf{N}_{2} & {\left[2 \mathbf{K}_{0}+\frac{1}{2} \mathbf{N}_{1}\right] \mathbf{a}} \\
\hline \text { Sym. } & \mathbf{a}^{\mathrm{T}} \mathbf{K}_{0} \mathbf{a}
\end{array}\right]
$$

Finally, introducing (51) in (25) and replacing the auxiliary variable $t$ by the unit value, the following is obtained:

with

$$
\begin{aligned}
U & =\frac{1}{12} \hat{\mathbf{a}}^{\mathrm{T}}\left[\hat{\mathbf{K}}_{\mathrm{T}}\right]_{t=1} \hat{\mathbf{a}} \\
\hat{\mathbf{f}} & =\frac{1}{3}\left[\hat{\mathbf{K}}_{\mathrm{T}}\right]_{t=1} \hat{\mathbf{a}} \\
\mathrm{d} \hat{\mathbf{f}} & =\left[\hat{\mathbf{K}}_{\mathrm{T}}\right]_{t=1} \mathrm{~d} \hat{\mathbf{a}}
\end{aligned}
$$

$$
\hat{\mathbf{a}}^{\mathrm{T}}=[\mathbf{a}, 1], \quad \mathrm{d} \hat{\mathbf{a}}^{\mathrm{T}}=[\mathrm{d} \mathbf{a}, 0]
$$

being $\hat{\mathbf{f}}$ and $\mathrm{d} \hat{\mathbf{f}}$ the extended vectors of nodal loads and their differential increments, to which a new component has been added in correspondence with the variable $t$ introduced in vectors â and dâ. This additional component can be eliminated before proceeding to the resolution of the equation system (55) without affecting the final result.

The simplification resulting from the replacement of the usual expressions (1) by the new ones (55) is obvious, and it means a drastic reduction of the computer memory needed to store the 
basic matrices. The auxiliary variable $t$ is the same for all the elements in the system, so that when the stiffness matrices of each element are assembled, the total number of degrees of freedom only increases by one, keeping, therefore, within the dimension of the problem.

\section{ILLUSTRATIVE EXAMPLE}

\section{2-d rod element}

Let us apply the method proposed to derive the secant and tangent stiffness matrices for the simple 2-d Euler-Bernouilli rod element. The same problem was also analysed in References 8 and (13).

Using an Updated Lagrangian Formulation (ULF) and a convective reference system Oxy attached to the beam cross sections with $x$ located along the beam axis, the only non-zero axial strain component is given by

$$
\boldsymbol{\varepsilon}=u_{, x}-z w_{, x x}+\frac{1}{2} w_{, x}^{2}
$$

where $z$ is the distance from a point to the beam axis and $u$ and $w$ are the longitudinal and transversal (deflection) displacements relative to the local axis Oxy.

The solution will be attempted following both the approach proposed by Rajasekaran and Murray $^{13}$ and the methodology presented in this paper.

Method of Rajasekaran and Murray. Starting from equation (57), this may be rewritten as (4a):

$$
\varepsilon=\mathbf{h}^{\mathrm{T}} \mathbf{g}+\frac{1}{2} \mathbf{g}^{\mathrm{T}} \mathbf{H g}
$$

in which $\mathbf{g}, \mathbf{h}$ and $\mathbf{H}$ denote the following matrices:

$$
\begin{aligned}
\mathbf{g}^{\mathrm{T}} & =\left[\begin{array}{lll}
u_{, x} & w_{, x} & w_{, x x}
\end{array}\right] \\
\mathbf{h}^{\mathrm{T}} & =\left[\begin{array}{lll}
1 & 0 & -z
\end{array}\right] \\
\mathbf{H} & =\left[\begin{array}{lll}
0 & 0 & 0 \\
0 & 1 & 0 \\
0 & 0 & 0
\end{array}\right]
\end{aligned}
$$

If displacement is discretized by choosing a standard $C_{0}$ continuous linear interpolation for axial displacement $u$, and a $C_{1}$ continuous cubic (Hermitian) one for transverse displacement $w$, we can write, for each individual element: ${ }^{15}$

$$
\mathbf{u}=\left\{\begin{array}{l}
u \\
w
\end{array}\right\}=\left[\begin{array}{cccccc}
N_{1} & 0 & 0 & N_{2} & 0 & 0 \\
0 & N_{3} & N_{5} & 0 & N_{4} & N_{6}
\end{array}\right]\left\{\begin{array}{c}
u_{1} \\
w_{1} \\
\left.\frac{\partial w}{\partial x}\right)_{1} \\
u_{2} \\
w_{2} \\
\left(\frac{\partial w}{\partial x}\right)_{2}
\end{array}\right\}=\mathbf{N a}^{(\mathrm{e})}
$$


with

$$
\begin{array}{ll}
N_{1}=1-\xi, & N_{2}=\xi \\
N_{3}=1-3 \xi^{2}+2 \xi^{3}, & N_{4}=3 \xi^{2}-2 \xi^{3} \\
N_{5}=L\left(1-2 \xi^{2}+\xi^{3}\right), & N_{6}=L\left(-\xi^{2}+\xi^{3}\right)
\end{array}
$$

where $\xi=x / L$ is a natural co-ordinate defined along the beam axis, and $L$ is the length of a standard beam element.

Matrix $\mathbf{P}$ — see equation (19) — can now be obtained:

$$
\mathbf{P}=\left[\begin{array}{cccccc}
\frac{-1}{L} & 0 & 0 & \frac{1}{L} & 0 & 0 \\
0 & \frac{6}{L}\left(-\xi+\xi^{2}\right) & \left(1-4 \xi+3 \xi^{2}\right) & 0 & \frac{6}{L}\left(\xi-\xi^{2}\right) & \left(-2 \xi+3 \xi^{2}\right) \\
0 & \frac{6}{L^{2}}(-1+2 \xi) & \frac{1}{L}(-4+6 \xi) & 0 & \frac{6}{L^{2}}(1-2 \xi) & \frac{1}{L}(-2+6 \xi)
\end{array}\right]
$$

The constitutive matrix $\mathbf{C}$ is reduced to the scalar $E A$. Introducing expressions (3) in (2) and integrating over the cross-section, we have

$$
\begin{aligned}
& \mathbf{L}_{0}=E \int_{A} \mathbf{h h}^{\mathrm{T}} \mathrm{d} A=\mathrm{EA}\left[\begin{array}{ccc}
1 & 0 & 0 \\
0 & 0 & 0 \\
0 & 0 & I / A
\end{array}\right] \\
& \mathbf{L}_{1}=E \int_{A}\left(\mathbf{h g}^{\mathrm{T}} \mathbf{H}+\mathbf{g}^{\mathrm{T}} \mathbf{h} \mathbf{H}+\mathbf{H} \mathbf{g h} \mathbf{T}^{\mathrm{T}}\right) \mathrm{d} A=E A\left[\begin{array}{ccc}
0 & w_{, x} & 0 \\
w_{, x} & u_{, x} & 0 \\
0 & 0 & 0
\end{array}\right] \\
& \mathbf{L}_{2}=E \int_{A}\left(\mathbf{H g g}^{\mathrm{T}} \mathbf{H}+\frac{1}{2}\left(\mathbf{g}^{\mathrm{T}} \mathbf{H g}\right) \mathbf{H}\right) \mathrm{d} A=\frac{3}{2} E A\left[\begin{array}{ccc}
0 & 0 & 0 \\
0 & w_{, x}^{2} & 0 \\
0 & 0 & 0
\end{array}\right]
\end{aligned}
$$

where $E, I$ and $A$ denote the modulus of elasticity, the moment of inertia and the cross-sectional area of the beam, respectively. The energy, secant and tangent stiffness matrices can now be obtained by equations (1a)-(1c).

Method proposed in this paper. The strain energy functional for a rod element is given by

$$
\Psi=\frac{1}{2} \int_{A} E \varepsilon^{2} \mathrm{~d} A=\frac{E A}{2}\left[u_{, x}^{2}+\lambda w_{, x x}^{2}+u_{, x} w_{, x}^{2}+\frac{1}{4} w_{, x}^{4}\right] \quad \text { with } \quad \lambda=I / A
$$


Identifying functions $p_{i}$ with the components of the displacement gradient vector (58) and using the general decomposition presented in equations (23), then

$$
\begin{aligned}
& \mathbf{p}^{\mathrm{T}}=\left[\begin{array}{lll}
p_{1} & p_{2} & p_{3}
\end{array}\right]=\left[\begin{array}{lll}
u_{, x} & w_{, x} & w_{, x x}
\end{array}\right] \\
& \Psi^{(2)}=\frac{1}{2} E A\left[u_{, x}^{2}+\lambda w_{, x x}^{2}\right] \\
& \Psi^{(3)}=\frac{1}{2} E A u_{, x} w_{, x}^{2} \\
& \Psi^{(4)}=\frac{1}{2} E A w_{, x}^{4}
\end{aligned}
$$

The elements of $\mathbf{B}^{(n)}$ matrices $(n=2,3,4)$ are obtained directly from equation (27), i.e.

$$
\mathbf{B}^{(2)}=E A\left[\begin{array}{lll}
1 & 0 & 0 \\
0 & 0 & 0 \\
0 & 0 & \lambda
\end{array}\right], \quad \mathbf{B}^{(3)}=E A\left[\begin{array}{ccc}
0 & w_{, x} & 0 \\
w_{, x} & u_{, x} & 0 \\
0 & 0 & 0
\end{array}\right], \quad \mathbf{B}^{(4)}=\frac{3}{2} E A\left[\begin{array}{ccc}
0 & 0 & 0 \\
0 & w_{, x}^{2} & 0 \\
0 & 0 & 0
\end{array}\right]
$$

If $\mathbf{B}^{(2)}, \mathbf{B}^{(3)}$ and $\mathbf{B}^{(4)}$ are identified with $\mathbf{L}_{0} \mathbf{L}_{1}$ and $\mathbf{L}_{2}$, then, the equivalence between both procedures is obvious. Note that matrix $\mathbf{P}$ is, in this case, also given by equation (63). Finally, these three matrices can be reduced to only one, as explained in the previous section. For this purpose, expression (47) is homogenized as:

$$
\boldsymbol{\varepsilon}=t\left(\mathbf{u}_{, x}-z \mathbf{w}_{, x x}\right)+\frac{1}{2}\left(\mathbf{w}_{, x}\right)^{2}
$$

which can also be written symbolically:

being now

$$
\boldsymbol{\varepsilon}=\frac{1}{2} \hat{\mathbf{g}}^{\mathrm{T}} \hat{\mathbf{H}} \hat{\mathbf{g}}
$$

$$
\hat{\mathbf{g}}^{\mathrm{T}}=\left[\mathbf{p}^{\mathrm{T}}, t\right]=\left[\begin{array}{llll}
u_{, x} & w_{, x} & w_{, x x} & t
\end{array}\right] \quad \hat{\mathbf{H}}=\left[\begin{array}{cccc}
0 & 0 & 0 & 1 \\
0 & 1 & 0 & 0 \\
0 & 0 & 0 & -z \\
1 & 0 & -z & 0
\end{array}\right]
$$

Applying (53) directly or replacing $\mathbf{H}$ by $\hat{\mathbf{H}}$ in (64c), we obtain

$$
\hat{\mathbf{B}}=E A\left[\begin{array}{ccc|c}
t^{2} & t \mathbf{w}_{, x} & 0 & 2 t \mathbf{u}_{, x}+\frac{1}{2} \mathbf{w}_{, x}^{2} \\
& t \mathbf{u}_{, x}+\frac{3}{2} \mathbf{w}_{, x}^{2} & 0 & \mathbf{w}_{, x} \mathbf{u}_{, x} \\
& & t^{2} \lambda & 2 t \lambda \mathbf{w}_{, x x} \\
\hline & \text { Sym. } & & u_{, x}^{2}+\lambda \mathbf{w}_{, x x}^{2}
\end{array}\right]
$$

Making now $t=1$, the result is - see equation (54):

$$
\hat{\mathbf{B}}=E A\left[\begin{array}{ccc|c}
1 & \mathbf{w}_{, x} & 0 & 2 \mathbf{u}_{, x}+\frac{1}{2} \mathbf{w}_{, x}^{2} \\
& \mathbf{u}_{, x}+\frac{3}{2} \mathbf{w}_{, x}^{2} & 0 & \mathbf{w}_{, x} \mathbf{u}_{, x} \\
& & \lambda & 2 \lambda \mathbf{w}_{, x x} \\
\hline \text { Sym. } & & u_{, x}^{2}+\lambda \mathbf{w}_{, x x}^{2}
\end{array}\right]
$$


The energy, secant and tangent stiffness matrices can now be simply obtained by equations (51). This classical example has shown the relative simplicity of the method proposed in this paper for deriving symmetric expressions for energy, secant and tangent stiffness matrices.

\section{CONCLUDING REMARKS}

The methodology proposed allows the derivation of a symmetric form for the energy, secant and tangent stiffness matrices in a general systematic way. This unified treatment may be applied to every structural finite element which accomplishes the two following constraints: (a) Internal energy may be written as in (30) and (31) (with $\mathbf{C}=$ constant), and (b) $p_{i}$ functions are linear on nodal displacements $a_{i}$, i.e., $\mathbf{P}$ is a constant matrix in (19). The ideas behind the approach proposed are very similar to those inherent to the Core-Congruential Formulation of Felippa et al. The coincidence of the general expressions with the classical particular forms derived in References 3, 5, 8, 11-14 clearly unifies the original methodologies and should be of academic as well as practical interest. This approach can also be very useful to derive the energy and secant stiffness matrices for structural instability analysis of rods, plates and shells following the ideas presented in References 6, 7,11 and 12. These possibilities are currently being investigated by the authors.

\section{APPENDIX}

\section{Homogeneous functions}

A function $F\left(x_{1}, x_{2}, \ldots, x_{r}\right)$ is called a homogeneous function of degree $k$ if it satisfies the following identity in $t$ :

$$
F\left(t x_{1}, t x_{2}, \ldots, t x_{r}\right) \equiv t^{k} F\left(x_{1}, x_{2}, \ldots, x_{r}\right)
$$

By differentiating both sides of (73) with respect to $t$, we obtain

$$
\begin{aligned}
& x_{i} F_{, x_{i}}\left(t x_{1}, t x_{2}, \ldots, t x_{r}\right) \equiv k t^{k-1} F\left(x_{1}, x_{2}, \ldots, x_{r}\right) \\
& \text { Sum on } i(1,2, \ldots, r)
\end{aligned}
$$

If we substitute $t=1$ in (74), we deduce the characteristic Euler's relation

$$
\begin{aligned}
& x_{i} F_{, x_{i}}\left(x_{1}, x_{2}, \ldots, x_{r}\right) \equiv k F\left(x_{1}, x_{2}, \ldots, x_{r}\right) \\
& \text { Sum on } i(1,2, \ldots, r)
\end{aligned}
$$

Conversely, it may be proved that not only the validity of (75) is a consequence of the homogeneity of the function $F\left(x_{1}, x_{2}, \ldots, x_{r}\right)$, but also that the homogeneity of the function is a consequence of Euler's relation, so that we can state Euler's theorem as follows: relation (75) is a necessary and sufficient condition for the homogeneity of the function.

It can be easily shown that every derivative $F_{, x_{i}}\left(x_{1}, x_{2}, \ldots, x_{r}\right)$ is also a homogeneous function of degree $k-1$, so that applying now Euler's theorem to these derivatives, we obtain

$$
\begin{aligned}
& x_{i} x_{j} F_{, x_{i} x_{j}}\left(x_{1}, x_{2}, \ldots, x_{r}\right) \equiv k(k-1) F\left(x_{1}, x_{2}, \ldots, x_{r}\right) \\
& \text { Sum on } i \text { and } j(1,2, \ldots, r)
\end{aligned}
$$

This result can be generalized for higher derivatives as follows:

$$
\underset{\text { Sum on the s indices } x_{i, j} \ldots l}{x_{i} x_{j} \ldots x_{1} F_{x_{i} x_{j}}}\left(x_{1}, x_{2}, \ldots, x_{r}\right) \equiv \frac{k !}{(k-s) !} F\left(x_{1}, x_{2}, \ldots, x_{r}\right)
$$




\section{REFERENCES}

1. E. Carrera, "Sull'uso dell'operatore secant in analisi non-lineare di strutture multistrato con il metodo degli elementi finiti', in ATTI, XI Congresso Nazionale AIMETA, Trento, 28 September-2 October 1992.

2. H. Duddeck, B. Kröplin, D. Dinkler, J. Hillmann and W. Wagenhuber, Non-linear Computations in Civil Engineering Structures (in German), DFG Colloquium, 2-3 March 1989, Springer, Berlin, 1989.

3. C. Felippa, 'Discussions on the paper by Rajasekaran and Murray (1973)', J. Struct. Div. ASCE, 100, 2521-2523 (1974).

4. C. Felippa and L. A. Crivelli, 'The core congruential formulation of geometrically non-linear finite elements', in P. Wriggers and W. Wagner (eds.), Non Linear Computational Mechanics. The State of the Art, Springer, Berlin, 1991.

5. C. Felippa, L. A. Crivelli and B. Haugen, 'A survey of the core congruential formulation for geometrically non-linear TL finite elements', Archives Comp. Meth. Engng., 1, 1-48 (1994).

6. B. Kröplin, D. Dinkler and J. Hillmann, 'An energy perturbation method applied to non-linear structural analysis', Comp. Meth. Appl. Mech. Engng., 52, 885-897 (1985).

7. B. Kröplin, 'Instability prediction by energy perturbation', in H. Alder, J. C. Heinrich, S. Lavanchy, E. Oñate and B. Suárez (eds.), Numerical Methods in Applied Sciences and Engineering, CIMNE, Barcelona, 1992.

8. R. Mallet and P. Marcal, 'Finite element analysis of non-linear structures', J. Struct. Div. ASCE, 14, 2081-2105 (1968).

9. E.Oñate, J. Oliver, J. Miquel and B. Suárez, 'Finite element formulation for geometrically non-linear problems using a secant matrix', in S. Atluri and G. Yagawa (eds.), Computational Plasticity '86, Springer, Berlin, 1986.

10. E. Oñate, 'Possibilities of the secant stiffness matrix for non-linear finite element analysis', in N. Bicanic et al. (eds.), Non-Linear Engineering Computation, Pineridge Press, Swansea.

11. E. Oñate, 'On the derivation of the secant stiffness matrix for non-linear finite element analysis', Comput. Mech., 15, 572-593 (1995).

12. E. Oñate and W. T. Matias, 'A critical displacement approach for predicting structural instability', in Comput. Meth. Appl. Mech. Engng., 134, 135-161 (1996).

13. S. Rajasekaran and D. W. Murray, 'On incremental finite element matrices', J. Struct. Div. ASCE, 99, 7423-7438 (1973).

14. R. D. Wood and B. Schrefler, 'Geometrically non-linear analysis - A correlation of finite element notations', Int. J. Numer. Meth. Engng., 12, 635-642 (1978).

15. O. C. Zienkiewicz and R. L. Taylor, The Finite Element Method, Vol. I, McGraw-Hill, New York, 1991.

16. M. Badawi and A. R. Cusens, 'Symmetry of the stiffness matrices for geometrically non-linear analysis', Commun. Appl. Numer. Meth., 8, 135-40 (1992). 